University of Wollongong

Research Online

Australian Institute for Innovative Materials -

Papers

Australian Institute for Innovative Materials

$1-1-2014$

Spin-cycloid instability as the origin of weak ferromagnetism in the disordered perovskite Bi0.8La0.2Fe0.5Mn0.503

J Bertinshaw

University of New South Wales

D L. Cortie

University of Wollongong, dlc422@uowmail.edu.au

Z X. Cheng

University of Wollongong, cheng@uow.edu.au

M Avdeev

ANSTO

A J. Studer

ANSTO

See next page for additional authors

Follow this and additional works at: https://ro.uow.edu.au/aiimpapers

Part of the Engineering Commons, and the Physical Sciences and Mathematics Commons

Research Online is the open access institutional repository for the University of Wollongong. For further information contact the UOW Library: research-pubs@uow.edu.au 


\title{
Spin-cycloid instability as the origin of weak ferromagnetism in the disordered perovskite BiO.8La0.2Fe0.5Mn0.5O3
}

\author{
Abstract \\ Powder neutron diffraction and magnetometry studies have been conducted to investigate the \\ crystallographic and magnetic structure of Bi0.8 $\mathrm{La} 0.2 \mathrm{Fe} 0.5 \mathrm{Mn} 0.503$. The compound stabilizes in the \\ Imma orthorhombic crystal symmetry in the measured temperature range of 5 to $380 \mathrm{~K}$, with a transition \\ to antiferromagnetic order at $\mathrm{TN} \approx 240 \mathrm{~K}$. The spin cycloid present for BiFeO3 is found to be absent with \\ $50 \% \mathrm{Mn} 3+$ cation substitution, leading to G-type antiferromagnetic order with an enhanced out-of-plane \\ canted ferromagnetic component, evident from measurable weak-ferromagnetic hysteresis. Structural \\ modifications do not solely explain this behavior, indicating that modified electron exchange interactions \\ must be taken into account. A classical spin simulation was developed to investigate the effect of \\ random substitution in a disordered pseudocubic perovskite. The calculations took into account the \\ nearest-neighbor, next-nearest-neighbor, and Dzyaloshinskii-Moriya interactions, along with the local spin \\ anisotropy. Using this framework to extend the established Hamiltonian model for BiFeO3, we show that \\ only certain types of perturbations at a magnetic defect and the surrounding molecular fields trigger a \\ simultaneous collapse of cycloidal order and the emergence of the long-range weak-ferromagnetic \\ component. By adopting values for the Mn molecular fields appropriate for REMnO3 (RE= rare earth), \\ simulations of BiMn0.5Fe0.503 exhibit the key magnetic properties of our experimental observations.

\section{Keywords} \\ 503 , origin, weak, ferromagnetism, disordered, perovskite, bi0, 8la0, spin, $2 \mathrm{fe} 0$, cycloid, $5 \mathrm{mn} 0$, instability

\section{Disciplines} \\ Engineering | Physical Sciences and Mathematics

\section{Publication Details} \\ Bertinshaw, J., Cortie, D. L., Cheng, Z. X., Ulrich, C., Avdeev, M., Studer, A. J., Klose, F. \& Wang, X. L. (2014). \\ Spin-cycloid instability as the origin of weak ferromagnetism in the disordered perovskite \\ Bi0.8La0.2Fe0.5Mn0.503. Physical Review B: Condensed Matter and Materials Physics, 89 (14), \\ 144422-1-144422-11.
}

\section{Authors}

J Bertinshaw, D L. Cortie, Z X. Cheng, M Avdeev, A J. Studer, F Klose, C Ulrich, and X L. Wang 


\title{
Spin-cycloid instability as the origin of weak ferromagnetism in the disordered perovskite $\mathrm{Bi}_{0.8} \mathrm{La}_{0.2} \mathrm{Fe}_{0.5} \mathrm{Mn}_{0.5} \mathrm{O}_{3}$
}

\author{
J. Bertinshaw, ${ }^{1,2}$ D. L. Cortie, $, 3,{ }^{3, *}$ Z. X. Cheng, ${ }^{3}$ M. Avdeev, ${ }^{2}$ A. J. Studer, ${ }^{2}$ F. Klose, ${ }^{2}$ C. Ulrich, ${ }^{1,2}$ and X. L. Wang ${ }^{3}$ \\ ${ }^{1}$ School of Physics, The University of New South Wales, Sydney, NSW 2052, Australia \\ ${ }^{2}$ Australian Nuclear Science and Technology Organisation, Lucas Heights, NSW 2234, Australia \\ ${ }^{3}$ The Institute for Superconducting and Electronic Materials, The University of Wollongong, Wollongong, NSW, 2522, Australia
}

(Received 7 November 2013; revised manuscript received 6 March 2014; published 23 April 2014)

\begin{abstract}
Powder neutron diffraction and magnetometry studies have been conducted to investigate the crystallographic and magnetic structure of $\mathrm{Bi}_{0.8} \mathrm{La}_{0.2} \mathrm{Fe}_{0.5} \mathrm{Mn}_{0.5} \mathrm{O}_{3}$. The compound stabilizes in the Imma orthorhombic crystal symmetry in the measured temperature range of 5 to $380 \mathrm{~K}$, with a transition to antiferromagnetic order at $T_{\mathrm{N}} \approx 240 \mathrm{~K}$. The spin cycloid present for $\mathrm{BiFeO}_{3}$ is found to be absent with $50 \% \mathrm{Mn}^{3+}$ cation substitution, leading to $G$-type antiferromagnetic order with an enhanced out-of-plane canted ferromagnetic component, evident from measurable weak-ferromagnetic hysteresis. Structural modifications do not solely explain this behavior, indicating that modified electron exchange interactions must be taken into account. A classical spin simulation was developed to investigate the effect of random substitution in a disordered pseudocubic perovskite. The calculations took into account the nearest-neighbor, next-nearest-neighbor, and Dzyaloshinskii-Moriya interactions, along with the local spin anisotropy. Using this framework to extend the established Hamiltonian model for $\mathrm{BiFeO}_{3}$, we show that only certain types of perturbations at a magnetic defect and the surrounding molecular fields trigger a simultaneous collapse of cycloidal order and the emergence of the long-range weak-ferromagnetic component. By adopting values for the $\mathrm{Mn}$ molecular fields appropriate for $R E \mathrm{MnO}_{3}(R E=$ rare earth), simulations of $\mathrm{BiMn}_{0.5} \mathrm{Fe}_{0.5} \mathrm{O}_{3}$ exhibit the key magnetic properties of our experimental observations.
\end{abstract}

DOI: 10.1103/PhysRevB.89.144422

PACS number(s): 61.05.fm

\section{INTRODUCTION}

Strategies to improve the magnetic and ferroelectric properties exhibited by the room-temperature multiferroic bismuth ferrite $\left(\mathrm{BiFeO}_{3}\right)$ have received a significant amount of attention in recent years, primarily for implementation in nextgeneration devices for information storage [1] and spintronic applications [2,3]. While the ferroelectric polarization can be controlled through an applied voltage, the magnetic degree of freedom in $\mathrm{BiFeO}_{3}$ is difficult to measure directly as it exhibits antiferromagnetic (AFM) order with a related incommensurate spin-cycloidal structure [4]. A promising avenue of research to induce a sizable ferromagnetic component necessary for technical applications is cation substitution [5-10], which has been shown to both establish measurable weak-ferromagnetic (wFM) behavior and enhance ferroelectric properties. However, it is only comparatively recently that the mechanisms responsible for the magnetic ground state of pure $\mathrm{BiFeO}_{3}$ have been investigated in detail, enabling a more systematic approach to improve the properties of $\mathrm{BiFeO}_{3}$ [11-14]. The current work discusses the effect of substitutional doping in $\mathrm{BiFeO}_{3}$ on the magnetic structure and properties through a combined experimental and calculation approach.

Pure $\mathrm{BiFeO}_{3}$ crystallizes in the rhombohedral perovskite structure (space group $R 3 c$ ) and exhibits spontaneous magnetic $\left(T_{\mathrm{N}}=643 \mathrm{~K}\right)$ and electric $\left(T_{C} \approx 1100 \mathrm{~K}\right)$ polarization well above room temperature [4]. The noncentrosymmetric $R 3 c$ space group describes an antiferrodistortive rotation of the $\mathrm{FeO}_{6}$ octahedra around the elongated pseudocubic [111] body diagonal, along the direction of the electric polarization propagation. $\mathrm{Fe}^{3+}$-ion spins couple strongly to the ferroelastic

*Now at the University of British Columbia; cortie@phas.ubc.ca domains induced by the rhombohedral structural distortion, establishing an easy magnetic plane which lies perpendicular to the octahedral tilt axis of rotation. Like $\mathrm{Fe}_{2} \mathrm{O}_{3}$ and other systems in the $R E \mathrm{FeO}_{3}$ series $\left(R E=\right.$ rare earth), $\mathrm{BiFeO}_{3}$ has the potential to exhibit an out-of-plane wFM component, which forms as a result of a frustrated canted commensurate spin configuration due to the Dzyaloshinskii-Moriya (DM) spin-orbit interaction [15-17]. In addition to $G$-type collinear AFM magnetic order, an incommensurate spin cycloid propagates in one of six energetically degenerate axes in the magnetic easy plane, with an extended propagation rotation of $620 \AA$ [18-20]. Problematic for technological applications, this cycloidal order effectively cancels any measurable wFM resulting from the spin canting mechanism, as well as the potential for linear magnetoelectric coupling [17].

The magnetic exchange interactions responsible for the cycloid formation, and the precise details of its structure, have remained an open topic for research since its discovery $[13,14,21]$. Despite this, recent developments focusing on a full theoretical description of the magnetoelectric structure of $\mathrm{BiFeO}_{3}$ have led to a successful model Hamiltonian, which has shown through theoretical simulations to reproduce the cycloid using effective energy parameters derived from inelastic neutron scattering and light scattering techniques $[11,12,22,23]$. These investigations indicate the spin cycloid arises from competing interactions between nearest-neighbor (NN) and next-nearest-neighbor (NNN) superexchange interactions between the Fe ions, mediated by oxygen anions, with the propagation direction stabilized by the DM interactions.

The determination of a theoretical description of the spin-cycloid mechanism represents a major step towards a systematic study of the underlying physics of processes that disrupt the cycloid, in order to realize a measurable and controllable wFM moment. Cation substitution of either 
the $\mathrm{Bi} \mathrm{A}$ site or $\mathrm{Fe} \mathrm{B}$ site affects both the electric and magnetic polarization components, indicating a complex array of competing interactions [6-9]. While experimentally it is known that the cycloid can be perturbed through modifications of the $\mathrm{BiFeO}_{3}$ chemical and structural properties via cation substitution or strain fields [24,25], it remains unclear as to what magnetic perturbations are required to drive the $\mathrm{BiFeO}_{3}$ system to a canted spin structure and release latent wFM.

In past work, we reported on ferroelectric and magnetic properties of similar $\mathrm{Bi}_{(1-x)} \mathrm{La}_{(x)} \mathrm{Fe}_{(1-y)} \mathrm{Mn}_{(y)} \mathrm{O}_{3}$ compounds $[7,26]$. Here, we present neutron diffraction measurements performed to elucidate the precise influence of cation substitution on the magnetic and structural properties of polycrystalline $\mathrm{Bi}_{0.8} \mathrm{La}_{0.2} \mathrm{Fe}_{0.5} \mathrm{Mn}_{0.5} \mathrm{O}_{3}$. Monte Carlo simulations were conducted to investigate the effect of local perturbations resulting from substitution on the spin Hamiltonian. Experimental observations indicate single phase growth of an orthorhombic perovskite structure with a measurable wFM component and increased magnetic susceptibility. Our theoretical simulations indicate these properties likely develop as a result of localized variance in the strength of the DM interaction and spin anisotropy relative to the superexchange bond, triggering the collapse of the cycloid and emergence of weak-ferromagnetic hysteresis.

\section{EXPERIMENT METHODS}

$\mathrm{Bi}_{0.8} \mathrm{La}_{0.2} \mathrm{Fe}_{0.5} \mathrm{Mn}_{0.5} \mathrm{O}_{3}$ polycrystalline samples were prepared by the solid-state reaction method from precursor powders $\mathrm{Bi}_{2} \mathrm{O}_{3}, \mathrm{La}_{2} \mathrm{O}_{3}, \mathrm{Fe}_{2} \mathrm{O}_{3}$, and $\mathrm{MnCO}_{3}$ with purities of $>99.99 \%$. A pellet was formed from starting materials mixed well in the nominal mole ratio and sintered at $800{ }^{\circ} \mathrm{C}$ in ambient atmosphere. It was then sintered a second time at $960{ }^{\circ} \mathrm{C}$ with an additional $2 \% \mathrm{Bi}_{2} \mathrm{O}_{3}$ after grinding and briquetting. $20 \%$ of isovalent $\mathrm{La}$ was introduced to stabilize the compound in a homogeneous $\mathrm{Bi}_{(1-x)} \mathrm{La}_{(x)} \mathrm{Fe}_{0.5} \mathrm{Mn}_{0.5} \mathrm{O}_{3}$ phase, as pure $\mathrm{BiFe}_{0.5} \mathrm{Mn}_{0.5} \mathrm{O}_{3}$ is thermodynamically unstable in bulk form at ambient pressure [27].

Magnetization measurements were performed using a commercial vibrating sample magnetometer (Quantum Design PPMS). X-ray diffraction (XRD) was conducted using a PANanalytical X'Pert diffractometer with a $\mathrm{Cu}-K \alpha$ source. Diffraction patterns were analyzed by performing a leastsquares Rietveld refinement, utilizing the FULLPROF software suite [28].

High-resolution powder neutron diffraction measurements were performed using the instrument ECHIDNA, located at the Bragg Institute, ANSTO, Sydney, Australia. ECHIDNA is equipped with $128{ }^{3} \mathrm{He}$ linear position-sensitive detectors that are scanned during data collection to produce high-resolution diffraction patterns. A Ge monochromator was aligned on the (331) reflection with a takeoff angle of $140^{\circ}$, selecting in a wavelength of $2.4395 \AA$ and an accessible $Q$ range of $0.2-5.1 \AA^{-1}$. Collimation ensured a minimum full width at half maximum (FWHM) resolution of $0.4^{\circ}$.

The high-intensity powder neutron diffractometer Wombat, located at the Bragg Institute, was used to conduct a temperature-dependency study of the magnetic and crystallographic structures. WOMBAT uses a static monolithic position-sensitive ${ }^{3} \mathrm{He}$ detector, which enables fast data collection times with little penalty to resolution. No collimation was used in order to maximize the detection rate. A vertically focused Ge monochromator was set to align on the (113) reflection, with a $90^{\circ}$ takeoff angle, selecting an incident wavelength of $2.41 \AA$ and an accessible $Q$ range of $0.5-4.9 \AA^{-1}$.

\section{EXPERIMENTAL RESULTS}

\section{A. Room-temperature structure}

High-resolution neutron diffraction was first conducted at $300 \mathrm{~K}$ on the ECHIDNA instrument, above the transition to long-range antiferromagnetic order at $T_{\mathrm{N}}=240 \mathrm{~K}$. Rietveld refinement of the neutron diffraction was performed concurrently with laboratory XRD data to ensure the high quality of the sample and determine the precise crystal symmetry. The Imma space group was determined to most accurately represent the crystal symmetry of $\mathrm{Bi}_{0.8} \mathrm{La}_{0.2} \mathrm{Fe}_{0.5} \mathrm{Mn}_{0.5} \mathrm{O}_{3}$. The final result of the refinement of the neutron and $\mathrm{x}$-ray data sets is presented in Fig. 1, and structural parameters and quality of the neutron diffraction analysis are listed in Table I. The atoms are located at the following Wyckoff positions: A-site $\mathrm{Bi} / \mathrm{La}$ at $4 e\left(0, \frac{1}{4}, z\right), \mathrm{B}$-site $\mathrm{Fe} / \mathrm{Mn}$ at $4 a(0,0,0), \mathrm{O} 1$ at $4 e$, and $\mathrm{O} 2$ at $8 g\left(\frac{1}{4}, \mathrm{y}, \frac{1}{4}\right)$. A minor $\beta-\mathrm{Bi}_{2} \mathrm{O}_{3}$ secondary phase was revealed by both $\mathrm{x}$-ray and neutron diffraction to

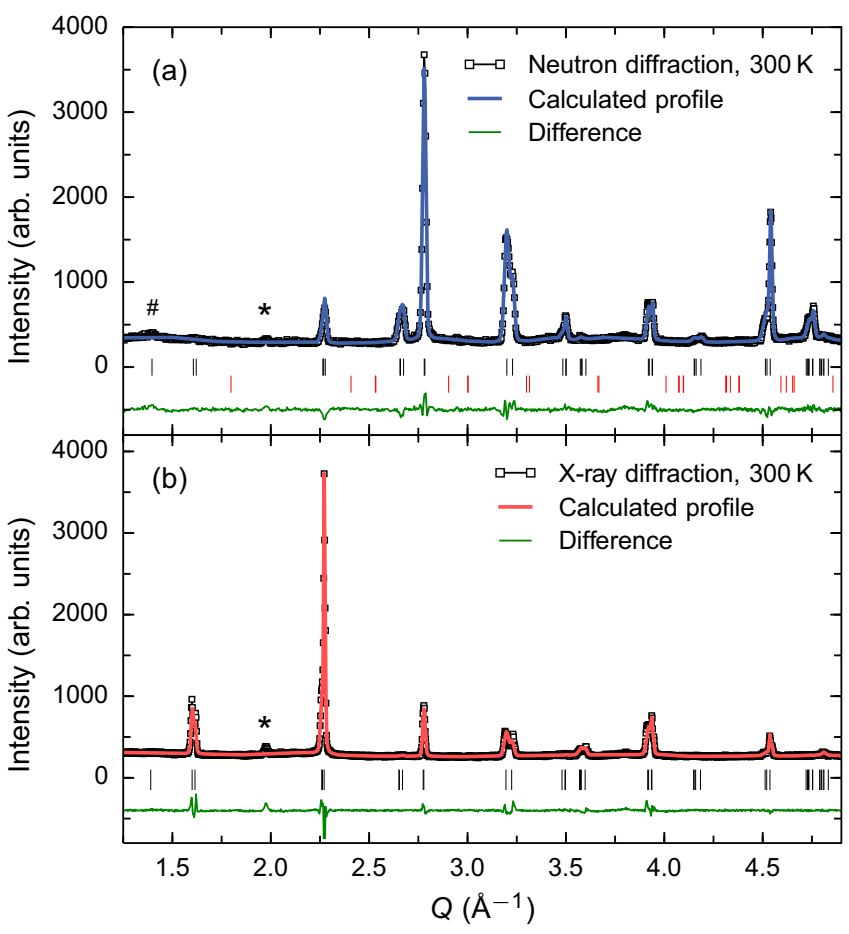

FIG. 1. (Color online) Refinement results of (a) high-resolution neutron and (b) laboratory x-ray diffraction performed at $T=300 \mathrm{~K}$. The system has been refined to the I $m m a$ crystal symmetry. Expected peak positions are indicated by vertical black markers. A small $\mathrm{Bi}_{2} \mathrm{O}_{3}$ impurity peak is marked with a star. The peak marked by a \# is attributed to $\sim 1 \%$ second-order neutron contamination. The red markers in (a) represent reflections corresponding to an additional in-phase $\mathrm{BO}_{6}$ rotation present in the related Pnma space group, none of which are apparent in the diffraction signal. 
TABLE I. Refinement results for high-resolution neutron diffraction performed at $300 \mathrm{~K}$. The structural (nuclear) phase residual is defined as $R_{\mathrm{N}}=\sum|| F_{\text {obs }}|-| F_{\text {cal }}|| / \sum\left|F_{\text {obs }}\right|$.

\begin{tabular}{lcc}
\hline \hline Temperature Space group & & $300 \mathrm{~K}$ Imma \\
\hline Lattice parameters & $a[\AA]$ & $5.5650(3)$ \\
& $b[\AA]$ & $7.8060(1)$ \\
Site coordinates & $c[\AA]$ & $5.5749(3)$ \\
& $z(\mathrm{Bi} / \mathrm{La})$ & $0.5023(9)$ \\
Bond distances & $y(\mathrm{O} 1)$ & $0.0734(8)$ \\
& $\mathrm{Mn} / \mathrm{Fe}-\mathrm{O} 1[\AA]$ & $0.5332(3)$ \\
Bond angles & $\mathrm{Mn} / \mathrm{Fe}-\mathrm{O} 2[\AA]$ & 1.994 \\
& $\mathrm{Mn} / \mathrm{Fe}-\mathrm{O} 1-\mathrm{Mn} / \mathrm{Fe}\left[{ }^{\circ}\right]$ & 1.987 \\
Statistical fit & $\mathrm{Mn} / \mathrm{Fe}-\mathrm{O} 2-\mathrm{Mn} / \mathrm{Fe}\left[{ }^{\circ}\right]$ & 156.3 \\
\hline \hline
\end{tabular}

be present in the compound, which is identified in Fig. 1 with a star. This is a result of the small amount of $\mathrm{Bi}_{2} \mathrm{O}_{3}$ added to compensate for evaporation of $\mathrm{Bi}$ during the second sinter at high temperatures. The peak marked by a \# in Fig. 1(a) is attributed to $\sim 1 \%$ second-order contamination in the neutron diffraction signal. Broadening of the diffraction reflections, above the instrumental resolution limits, indicated a measurable level of anisotropic microstrain present in the compound. It is likely this strain is a consequence of the random inhomogeneous distribution of $\mathrm{Mn}$ ions on the $\mathrm{Fe}$ $\mathrm{B}$ site and La-ions on the $\mathrm{Bi} \mathrm{A}$ site. In order to achieve an acceptable fit to the diffraction data, an anisotropic strain peak broadening model (quartic form) was implemented.

In order to determine the precise crystallographic structure, we have examined potential crystal symmetries most closely related to the rhombohedral ferroelectric $(R 3 c)$ and orthorhombic paraelectric (Pnma) phases of $\mathrm{BiFeO}_{3}$ [29]. The symmetry of the $A B \mathrm{O}_{3}$ perovskite family can be defined in terms of the nature of the $B_{6}$ octahedral rotations, due to misfit between the size of the A- and B-site ions (often described using Glazer tilt system notation [30]). While the $R 3 c$ space group $\left(a^{-} a^{-} a^{-}\right)$possesses identical antiferrodistortive (out-of-phase) tilts, Pnma $\left(a^{+} b^{-} b^{-}\right)$features an out-of-phase tilt for two axes, with an additional in-phase tilt. Lowering of symmetry results in the emergence of additional refections in the diffraction signal. No evidence of additional diffraction peaks corresponding to the in-phase octahedral tilt present in Pnma was found [shown as red markers in Fig. 1(a)], identifiying Imma $\left(a^{0} b^{-} b^{-}\right)$as the likely candidate for the crystal symmetry.

An important factor to consider in the competing interactions that give rise to a canted spin configuration and the strength of out-of-plane wFM is the influence of the rotation of the oxygen octahedra. Spin canting has previously been modeled in $\mathrm{BiFeO}_{3}$ for the Pnma and $R 3 c$ space groups [11]. From the results of this investigation, the direction of the spin canting angle is only dependent on the antiferrodistortive out-of-phase oxygen octahedral rotations present in both Imma and Pnma symmetries since these influence the nontrivial staggered components of the resulting DM vectors (see Sec. IV). The antiferrodistortive tilting of the octahedra is described by rotating the oxygen octahedra around the diad axis of the central B-site ion. Using the method described by Kennedy et al. in Ref. [31], an accurate value for the tilt angle of Imma was determined from the displacement $w$ of the $\mathrm{O} 2$ oxygen atoms from their cubic perovskite positions such that $\left(\frac{1}{4}, 0, \frac{1}{4}\right) \rightarrow\left(\frac{1}{4}, \frac{1}{2}-w, \frac{1}{4}\right)$. The out-of-phase tilt is then given by $\tan \phi=4 \sqrt{2} w$. From our neutron diffraction results, $\phi=10.6(1)^{\circ}$. Previous diffraction measurements on $\mathrm{BiFeO}_{3}$ revealed an oxygen octahedral rotation angle of $10.6^{\circ}$ [32]. Importantly, the wFM canting angle is thought to be linearly related to the rotation angles in both Pnma and $R 3 c$ space groups up to $\sim 11^{\circ}$ [11]. In the present case, given the very similar rotational angle, it is unlikely that the increased wFM moment arises from an increased octahedral rotation, as reported in previous studies of doped $\mathrm{BiFeO}_{3}$ compounds $[7,33]$. Instead, the increase in wFM from expected latent values of pure $\mathrm{BiFeO}_{3}$ are likely to arise from modifications to the relative strength of competing DM interactions and anisotropy compared to the antiferromagnetic superexchange. This is investigated in detail through Monte Carlo simulations in Sec. IV.

\section{B. Magnetic properties}

The magnetometry results in Fig. 2 demonstrate that the compound exhibits a measurable weak-ferromagnetic signal, which results from a frustrated canted magnetic structure similar to the case known for $\mathrm{BiMn}_{0.5} \mathrm{Fe}_{0.5} \mathrm{O}_{3}$ thin films [27]. The hysteresis shown in Fig. 2(a) indicates a relatively small canting component in the AFM structure below $T_{\mathrm{N}}$, with a maximum moment of $0.5 \mu_{\mathrm{B}} /$ f.u. at a field of 5 T. From Fig. 2(b), the Néel transition to $G$-type antiferromagnetic order was found to occur at $T_{\mathrm{N}}=240 \mathrm{~K}$. The difference between fieldcooled and zero-field-cooled magnetization measurements below $50 \mathrm{~K}$ is likely due to the freezing of antiferromagnetic domains. The Curie-Weiss temperature was determined from the high- $T$ paramagnetic data to be $\sim \theta=-245 \mathrm{~K}$, which corresponds well with $T_{\mathrm{N}}$, indicating no significant spin frustration in the system, despite the canted spin structure and relatively large amount of Mn substitution on the Fe-ion site.

The evolution of the magnetic structure was investigated by neutron diffraction in a temperature-dependency study from 5 to $360 \mathrm{~K}$ in $5-\mathrm{K}$ steps, using the instrument Wombat. 22 magnetic reflections were identified in the measured angular range, with the primary intensity at the $\left(\frac{1}{2} \frac{1}{2} \frac{1}{2}\right)_{\mathrm{pc}} /(110)_{\text {orth }}$ reflection. In Fig. 3, the diffraction pattern measured at $5 \mathrm{~K}$ is presented, which shows the majority of the magnetic signal emerges as a single peak at low $Q$. This peak is notated as the (101) in the orthorhombic representation and equivalent to the half-order $\left(\frac{1}{2} \frac{1}{2} \frac{1}{2}\right)_{\mathrm{pc}}$ pseudocubic peak, indicating a collinear $G$-type antiferromagnetic structure.

In undoped $\mathrm{BiFeO}_{3}$, for an electric polarization vector along the $[111]_{\mathrm{pc}}$ diagonal, a spin cycloid propagates with a length of $620 \AA$ in one of three equivalent perpendicular directions: $[10 \overline{1}]_{\mathrm{pc}},[011]_{\mathrm{pc}}$, or $[\overline{1} 10]_{\mathrm{pc}}$. Such a spin configuration would result in peak splitting of the Imma (101) peak along one of these three vector directions. These satellite peaks can been measured directly by neutron single crystal or powder diffraction, with an incommensurate splitting in reciprocal 

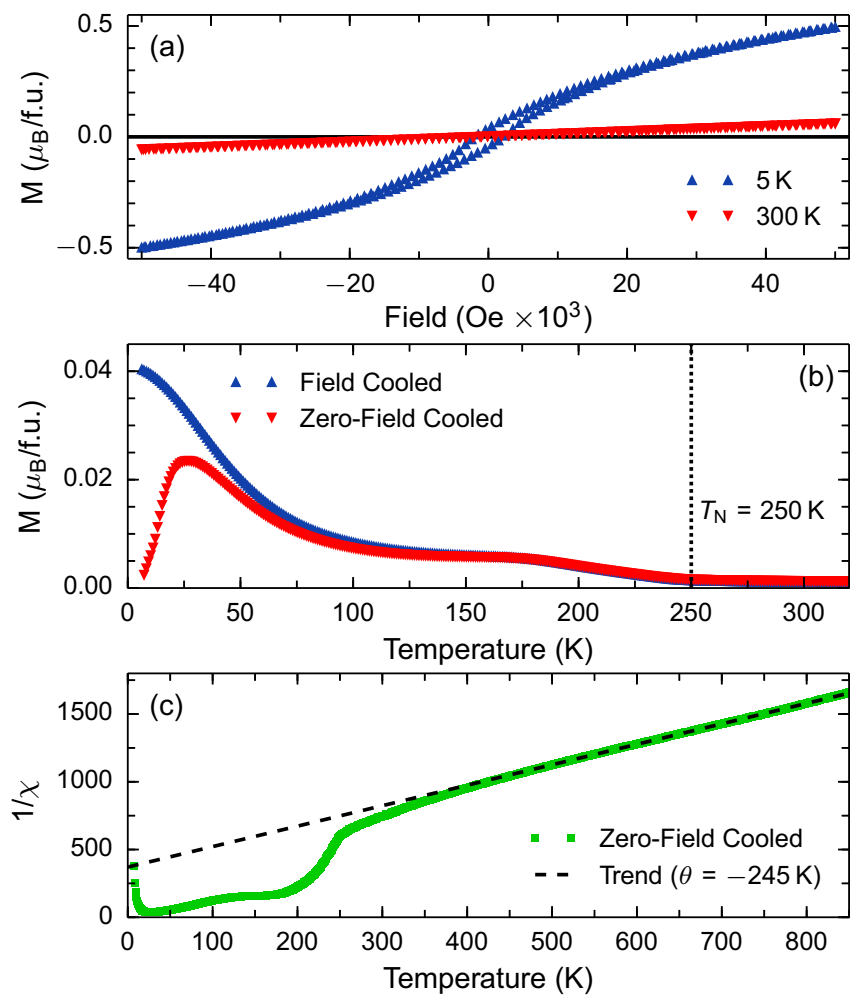

FIG. 2. (Color online) (a) Magnetic hysteresis measurements reveal a measurable weak ferromagnetic moment. (b) Field-cooled and zero-field-cooled temperature-dependency measurements show the transition to magnetic order occurs below $T_{\mathrm{N}}=240 \mathrm{~K}$. (c) The magnetic susceptibility indicates primarily antiferromagnetic order, with a negative intercept determined by fitting the Curie-Weiss law in the paramagnetic region.

lattice units of $\delta=0.005$ r.l.u. [19]. The expected additional satellite reflections would be visible within the resolution limits of the instrument Wombat used in this study, and are simulated as blue dashed lines in the inset of Fig. 3. The resolution function, as derived using the Thompson-HastingsCox profile function [34] for Rietveld refinement, gives a calculated FWHM of the magnetic peak, below the expected separation of the magnetic peak resulting from the cycloid present in undoped $\mathrm{BiFeO}_{3}$. Our peak-width analysis of the single resolvable magnetic Bragg peak forming below the $T_{\mathrm{N}}$ places an upper detection limit on the cycloid propagation length at $\sim 1500 \AA$, which is more than double to that known for $\mathrm{BiFeO}_{3}$. Together with weak-ferromagnetic hysteresis, our neutron result strongly indicates that no similar spin cycloid is present in the disorded $\mathrm{Bi}_{0.8} \mathrm{La}_{0.2} \mathrm{Fe}_{0.5} \mathrm{Mn}_{0.5} \mathrm{O}_{3}$ compound. This result confirms previous investigations of doped $\mathrm{BiFeO}_{3}$, which have reported an elongation and eventual collapse of the cycloid with increased doping on the A and/or B sites $[5,35,36]$.

As no additional nuclear reflections were found to form in the measured temperature range, the full temperaturedependency data set was refined with the structural phase fitted to the Imma space group. Initial structural parameters were set based upon the final results of the prior high-resolution refinement. Instrumental parameters were fitted for the $5-\mathrm{K}$

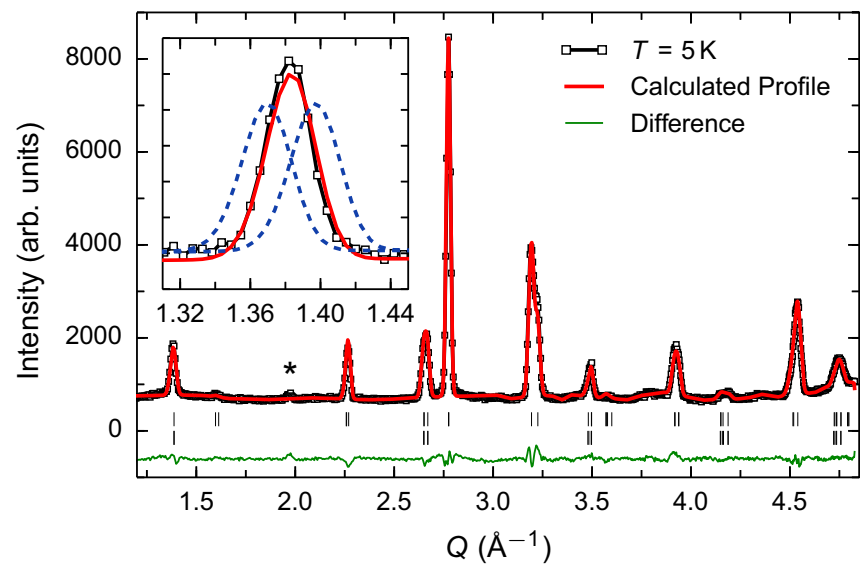

FIG. 3. (Color online) Rietveld refinement of neutron diffraction data collected at $5 \mathrm{~K}$. Inset: A closer examination of the (101) half-order magnetic reflection reveals no satellite peaks, indicating a collapse of the cycloid spin structure. The expected positions of additional peaks due to $\mathrm{BiFeO}_{3}$-type spin cycloid are shown as blue dashed lines. A small $\mathrm{Bi}_{2} \mathrm{O}_{3}$ impurity peak is marked with a star.

data set and fixed for succeeding temperature refinements. The temperature dependency of the lattice parameters and magnetic moment as determined by the Rietveld refinement are presented in Fig. 4 and are quite consistent with the values reported previously for $\mathrm{BiFeO}_{3}$ doped at the same levels of our sample [36]. The orthorhombic lattice parameter trends show no deviation from the behavior of a normal anharmonic solid, indicating the crystal structure remains stable in the Imma symmetry down to lowest temperatures. The magnetic structure was determined to exhibit collinear $G$-type antiferromagnetic order on the $\mathrm{Mn} / \mathrm{Fe}$ site, analyzed using the SARAH software package [37]. The spin canting readily evident in our magnetometry results in Fig. 2 should result in the emergence of additional magnetic peaks in the diffraction pattern, corresponding to a ferromagnetic moment
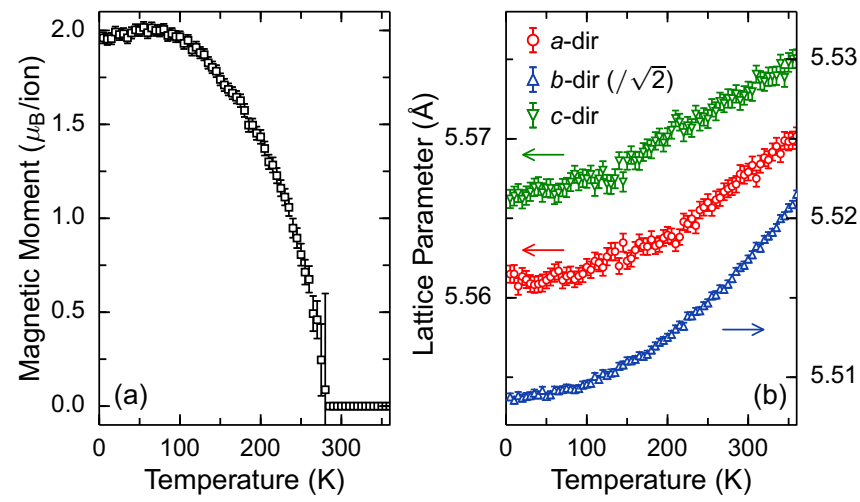

FIG. 4. (Color online) Rietveld refinement results of the neutron diffraction temperature-dependency study performed from 5 to $350 \mathrm{~K}$. (a) The magnetic moment of the $\mathrm{Fe} / \mathrm{Mn}$ follows a general trend from $T_{\mathrm{N}} \approx 240 \mathrm{~K}$ to a maximum of $2 \mu_{\mathrm{B}} /$ ion at low temperatures. (b) Orthorhombic lattice parameters indicate no deviation from the behavior of a normal anharmonic solid. The $b$-direction pseudocubic equivalent $b *=b / \sqrt{2}$ is plotted for legibility of all three crystal axes. 

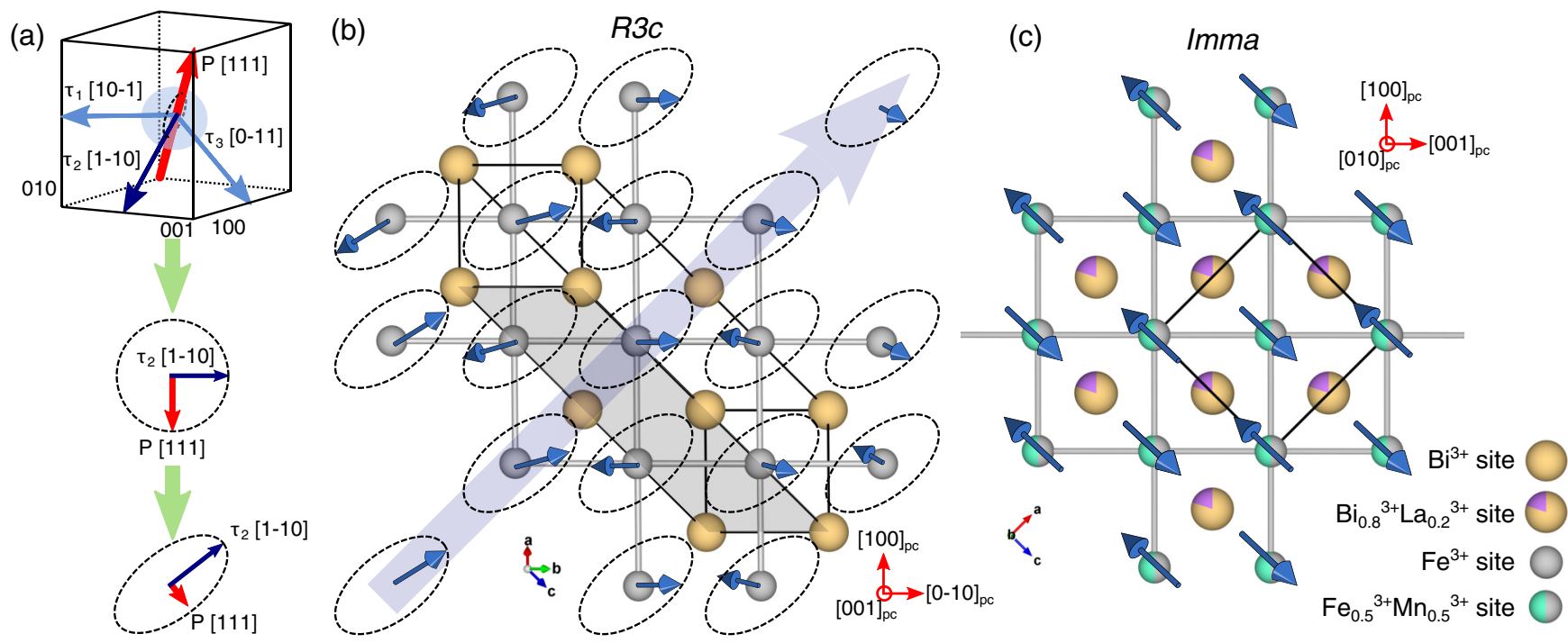

FIG. 5. (Color online) (a) An illustration of the relevant pseudocubic directions rotated into the frame of reference, and a determination of one of the planes in which the spins rotate, formed by the electric polarization $(P)$ and cycloid propagation vector $\left(\tau_{2}\right)$. The crystal and magnetic structures of (b) $\mathrm{BiFeO}_{3}(R 3 c)$ and (c) $\mathrm{Bi}_{0.8} \mathrm{La}_{0.2} \mathrm{Fe}_{0.5} \mathrm{Mn}_{0.5} \mathrm{O}_{3}$ (Imma) are visualized in a pseudocubic plane (O atoms are not shown). The red axes show the pseudocubic directions, whereas the colored axes represent the respective rhombohedral and orthorhombic cells. While both systems are well described by a pseudocubic cell, the cycloidal rotation of the spin moments present in $\mathrm{BiFeO}_{3}$ was not detected in $\mathrm{Bi}_{0.8} \mathrm{La}_{0.2} \mathrm{Fe}_{0.5} \mathrm{Mn}_{0.5} \mathrm{O}_{3}$, where the results are consistent with a uniform canted structure along a high-symmetry direction.

in a single plane, most significantly as a magnetic signal at the (030) reflection. However, the relatively small canting angle and size of the wFM moment under zero field as determined by the respective high-resolution neutron diffraction and magnetometry studies indicate such a signal is below the detection limits of the powder neutron diffraction technique, and indeed, no magnetic signal was detectable at the $(030)$ reflection. Based upon the collinear magnetic structure, the antiferromagnetic sublattice was determined to reveal a maximum moment at low temperatures of $\sim 2.0 \mu_{\mathrm{B}} /$ ion, whereas the magnetometry shows that the canted moment was $<0.1 \mu_{\mathrm{B}} /$ ion under zero field, increasing to $\sim 0.5 \mu_{\mathrm{B}} /$ ion at $5 \mathrm{~T}$. Figure 5 presents a simplified schematic of the differences in the chemical and magnetic structure of $\mathrm{BiFeO}_{3}$ and $\mathrm{Bi}_{0.8} \mathrm{La}_{0.2} \mathrm{Fe}_{0.5} \mathrm{Mn}_{0.5} \mathrm{O}_{3}$, illustrating the collapse of the cycloid.

\section{MODEL-SYSTEM BEHAVIOR}

The goal of this section is to interpret the experimental results in light of a classical spin model system implemented in a three-dimensional (3D) Monte Carlo computer simulation. In our theoretical approach, we developed a general model spin Hamiltonian simulating the magnetic properties of $\mathrm{BiFeO}_{3}$ in order to determine what perturbation(s) are required to explain the key experimental results: (1) suppression of the cycloid, (2) reduction of critical temperature, and (3) onset of a weakferromagnetic hysteresis.

\section{A. Atomistic model setup}

The general form of the model spin Hamiltonian appropriate for $\mathrm{BiFeO}_{3}$ was recently confirmed to be similar to those describing related structures such as $R E \mathrm{MnO}_{3}$ and

$$
\begin{aligned}
\alpha-\mathrm{Fe}_{2} \mathrm{O}_{3} & {[11,13,14]: } \\
H_{M}= & -\sum_{i j} J_{A B} \vec{S}_{i} \cdot \vec{S}_{j}-\sum_{i j} J_{A B}^{\prime} \vec{S}_{i} \cdot \vec{S}_{j}-\sum_{i} \kappa_{A} S_{i 111}^{2} \\
& -\sum_{i j} D_{A B} \vec{d}_{i j} \cdot\left(\vec{S}_{i} \times \vec{S}_{j}\right)-\mu_{b} \sum_{i} g_{A} \vec{B}_{\mathrm{ext}} \cdot \vec{S}_{i}
\end{aligned}
$$

In the Hamiltonian given in (1), the summations $\sum_{i}$ run over all atoms in a three-dimensional simple cubic lattice consisting of the B-site spin vectors, and terms with summations such as $\sum_{i j}$ also include the nearest or next-nearest neighbors. The first term describes a nearest-neighbor isotropic superexchange on a cubic lattice with an antiferromagnetic exchange constant given by $J_{A B}$. The subscripts specify the coupling appropriate to atom type of spin $i$ and spin $j$, required to consider the possibility of $\mathrm{Fe}-\mathrm{Fe}, \mathrm{Fe}-\mathrm{Mn}$, and $\mathrm{Mn}-\mathrm{Mn}$ pairs. The second term refers to a next-nearest-neighbor exchange between neighbors along a cubic diagonal with an antiferromagnetic coupling constant $J^{\prime}$. The third term describes a uniaxial spin anisotropy which prefers to maximize the spin component $S_{i 111}=\left|S_{i x}+S_{i y}+S_{i z}\right|$ along the unique elongated $[111]_{\mathrm{pc}}$ pseudocubic direction corresponding to the [001] direction in orthorhombic notation [13,14]. The fourth term describes the relativistic, antisymmetric superexchange of Dzyaloshinskii-Moriya form where the local DM vector governing the interaction between two spins is $d_{i j}$. The final term is the Zeeman interaction describing the coupling to the externally applied field where the effective moment is $g_{A} S_{i} \mu_{B}$ in a simple classical approach. The absolute values of the energy parameters $\left(J, J^{\prime}, D\right.$, and $\left.\kappa\right)$ for $\mathrm{BiFeO}_{3}$ were recently determined in two independent inelastic neutron scattering experiments and calculated using density functional theory (DFT) based on the local spin density approximation with 
TABLE II. Energies for the terms in the spin Hamiltonian of $\mathrm{BiFeO}_{3}$ determined from previously reported inelastic neutron scattering fits and DFT calculations. The bracketed terms show the ratio of the respective energy in units of the respective $\left|J_{\mathrm{Fe}-\mathrm{Fe}}\right|$. Energy ratios employed in the present simulation are listed in the last column, where values of $J^{\prime}, \kappa, D$ are increased by $\sim 5$ from the experimental ratios to reduce the cycloid length for the sake of computational efficiency.

\begin{tabular}{lcccc}
\hline \hline Parameter & Experiment 1 (meV) [14] & Experiment 2 (meV) [13] & DFT calculations (meV) [11] & This study \\
\hline$J_{\mathrm{Fe}-\mathrm{Fe}}$ & $-4.38(-1.0)$ & $-6.48(-1.0)$ & $-5.96(-1.0)$ & $(-1.0)$ \\
$J_{\mathrm{Fe}-\mathrm{Fe}}^{\prime}$ & $-0.15(-0.034)$ & $-0.29(-0.045)$ & $(-0.18)$ \\
$\kappa_{\mathrm{Fe}}$ & $<0.01(<0.002)$ & $0.0068(0.001)$ & $0.004-0.5(0.001-0.08)$ & $(0.08)$ \\
$D_{\mathrm{Fe}-\mathrm{Fe}}$ & $0.107(0.024)$ & $0.162(0.025)$ & $0.144(0.024)$ & $(0.144)$ \\
\hline \hline
\end{tabular}

an additional Hubbard energy (LSDA $+U$ ) $[11,13,14]$. The results are summarized in Table II. Although the two inelastic neutron experiments assumed different magnetic moments per $\mathrm{Fe}$, it is clear that the ratios between $J^{\prime} / J, D / J, \kappa / J$ (in brackets) for the pseudocubic case agree well, indicating that the ratio ultimately determines the zero-field ground state. While previous work using Monte Carlo methods showed that these ratios acceptably reproduce the ground-state magnetic structure and spin-wave dispersion [13], so far there has been no calculation showing whether weak ferromagnetism is supported in this scenario. As a magnetoelectric multiferroic, the wFM and cycloid propagation have previously shown to be affected by substitution of $\mathrm{La}$ and $\mathrm{Mn}$ on the respective $\mathrm{A}$ and $\mathrm{B}$ sites, or a combination of both $[35,38,39]$. The nature of the impact of A-site doping upon the ferroelectric, crystal, and magnetic properties of $\mathrm{La}_{x} \mathrm{Bi}_{1-x} \mathrm{MnO}_{3}$ is debated, a disparity potentially due to the difficulty in growing phase pure samples [40]. On the other hand, the magnetism of $\mathrm{La}_{x} \mathrm{Bi}_{1-x} \mathrm{Fe}_{0.5} \mathrm{Mn}_{0.5} \mathrm{O}_{3}$ as reported recently [36] is relatively insensitive to doping of the A-site cation, and the transition temperature, ordered spin structure, and susceptibility are fairly similar for the range of La concentrations $x=0.1-$ 0.5 [36]. Therefore, in the current simulation, we focus primarily on the magnetic effect of the B-site defect, although it is understood that the nonmagnetic A-site modification will indirectly contribute effects to the magnetic defect population. For this study, a computer simulation of the finite-temperature spin structure and susceptibility was implemented using a thermal Monte Carlo method where spins were treated as classical vectors $[41,42]$ and obey the extended Heisenberg model described by Eq. (1). The trial step used was a small rotation of the spin around the unit sphere. A standard heat-bath algorithm was used to accept the trial step and simulate Glauber dynamics [42]. While not a full electronic description of the system, the advantage of this well-established approach is that it provides a route to accurately model entropy, rather than only internal energy, and allows for the study of complex spin structures at finite temperature that are beyond the scope of simple analytical treatments or the scales possible with DFT calculations [43].

In our simple ionic model we assume that the total spin moment of $\mathrm{Fe}^{3+}$ is $\left|\overrightarrow{S_{\mathrm{Fe}}}\right|=2.5$ while that of the dopant $\left(\mathrm{Mn}^{3+}\right)$ is $\left|\overrightarrow{S_{\mathrm{Mn}}}\right|=2.0$. Previous $\mathrm{x}$-ray photoemission spectra and core-level DFT calculations have established both ions predominantly exist in the $3+$ state in $\mathrm{BiMn}_{0.5} \mathrm{Fe}_{0.5} \mathrm{O}_{3}$ [44]. DFT calculations in the $R 3$ group for $\mathrm{BiFeO}_{3}$ found that the local microscopic DM vectors are staggered in such a way that the net DM vector points along one of the (111) directions and has a magnitude that is specified by only two parameters $\alpha$ and $\beta$. Following a similar formalism to that used in the simulations of $R E \mathrm{MnO}_{3}$ [23], we express the local DM vectors as

$$
\begin{aligned}
& d_{i j}^{x}=\left(\begin{array}{l}
(-1)^{x+y+z} \alpha \\
(-1)^{x+y+z} \alpha \\
(-1)^{x+y+z} \beta
\end{array}\right), \quad d_{i j}^{y}=\left(\begin{array}{l}
(-1)^{x+y+z} \alpha \\
(-1)^{x+y+z} \beta \\
(-1)^{x+y+z} \alpha
\end{array}\right), \\
& d_{i j}^{z}=\left(\begin{array}{l}
(-1)^{x+y+z} \beta \\
(-1)^{x+y+z} \alpha \\
(-1)^{x+y+z} \alpha
\end{array}\right),
\end{aligned}
$$

where $d_{i j}^{\sigma}$ refers to the nearest-neighbor contact between atoms $i$ and $j$ in the cubic direction given by the superscript $\sigma=$ $x, y, z$. The parameters of $\alpha$ and $\beta$ were selected based closely on the DFT for $\mathrm{BiFeO}_{3}$ by Weingart et al. in Ref. [11], which matches well with experimental results (see Table II). In this study, the values of $\alpha=1$ and $\beta=1.2$ are fixed, expressing the experimental finding that the relevant oxygen rotation angles are nearly constant, however, we do allow modifications to the energy scale of the resulting vector controlled with an additional scalar multiplier $D$. It is important to note that the anticommutativity of the vector product on a canted antiferromagnetic lattice results in the property $\vec{S}_{i} \times \vec{S}_{j}=-\vec{S}_{j} \times \vec{S}_{i}$, but from a physical perspective, the energy of the microscopic DM spin-spin interaction for two spins needs to be independent of the order in which the mathematical operation is performed. While typically this is enforced with the microscopic condition that $d_{i j}=-d_{j i}$ [23], in this work the spin interactions are sorted by predefined array indices which achieve the same result. To mimic $\mathrm{Mn}$ inclusion with no site preference, the software randomly positions secondary ions throughout the lattice with a probability equal to the concentration (i.e., $x=0.5$ ), such that the local molecular field parameters need to be specified separately for the Mn-Mn, Fe-Fe, and Mn-Fe NN pairs. Statistically, the resulting microstructural layout of $\mathrm{Mn}$ in the lattice varies such that each initialization of the system produces slightly different layouts of $\mathrm{Fe}-\mathrm{Fe}$ and $\mathrm{Mn}-\mathrm{Mn}$ rich regions. For this reason, when $\mathrm{Mn}$ doping was included in the model, the results were averaged over five microstructural realizations to ensure that the conclusions were robust.

Throughout later sections, the reduced temperature $t$ is expressed in natural units of the strongest exchange energy $\left(\left|J_{\mathrm{Fe}-\mathrm{Fe}}\right|\right)$ such that $t=k_{B} T /\left|J_{\mathrm{Fe}-\mathrm{Fe}}\right|$ and the field is written in reduced units scaled to the main exchange parameter $\left(b_{\text {ext }}=\right.$ $\left.g_{A} \mu_{b} B_{\text {ext }} /\left|J_{\mathrm{Fe}-\mathrm{Fe}}\right|\right)$. To calculate the magnetic ground state, the virtual sample is zero-field cooled from the paramagnetic state at $t=20.1$ to 0.10 in steps of $\Delta t=0.5$ allowing for $2 \times 10^{3}$ Monte Carlo steps (MCs) at each temperature, before 
discarding a further $1 \times 10^{4}$ to $1 \times 10^{6} \mathrm{MCs}$ at the lowest temperature, and extracting the thermal averages. The software outputs a real-space 3D spin structure, along with the $3 \mathrm{D}$ Fourier transform of the spin-correlation functions $F$ :

$$
F_{\sigma}\left(\vec{S}, k_{B} T, \vec{k}\right)=\sum_{i j}\left\langle S_{i \sigma} S_{j \sigma}\right\rangle \cos \left[2 \pi \vec{k} \cdot\left(\vec{r}_{i}-\vec{r}_{j}\right)\right],
$$

where $\vec{k}=(h \hat{x}+k \hat{y}+l \hat{z})$ and $S_{i \sigma}$ refers to the spin component of the $i$ th spin along a cubic direction $\sigma=\hat{x} / \hat{y} / \hat{z}$. To estimate the magnetic transition temperature, the virtual sample is initiated in the ground state, and systematically heated in steps of $\Delta t=0.5$, taking $5 \times 10^{3}$ MCs per step in an applied field along the [1 10$]$ direction. At each temperature, the software extracts the antiferromagnetic order parameter corresponding to the sublattice magnetization as well as the average magnetization over both sublattices. To simulate the hysteresis, the sample is first stabilized in the ground state, and the applied field is swept between $b_{\text {ext }}=6$ to -6 in steps of 0.12 taking $10^{3}$ MCs per step, where the field is applied along the weak ferromagnetic easy axis in the (110) direction. Typically, each hysteresis includes $5 \times 10^{5}$ MCs per realization. Previous work has shown that experimental parameters produce a cycloid of length $\sim 100$ lattice constants, however, this length scale prohibits the usage of time-consuming atomistic simulations [12]. For this reason, the parameters were modified as to retain the approximate ratios of $D / \kappa / J^{\prime}$ but to reduce the ratio of $D / J$ in order to shrink the cycloid to a more manageable length scale (18 spins) [12]. While sacrificing a modicum of realism, this procedure allows for a more thorough exploration of parameter space to establish crucial trends and to calculate magnetic susceptibility for an $18 \times 18 \times 12$ 3D lattice with standard periodic boundary conditions. A range of different simulation sizes including $8 \times 8 \times 12$ to $48 \times 48 \times 48$ were tested to ensure our results were not caused by additional finite-size effects associated with expansion of the cycloid beyond the allowed size.

\section{B. Undoped case behavior}

To begin with the simpler case of uniform molecular fields, Fig. 6 shows the primary results for the model approximating the behavior of un-doped $\mathrm{BiFeO}_{3}$ using just the parameters listed in Table II. In Figs. 6(b)-6(d), the real-space line profile and correlation functions are plotted showing a periodic real-space structure giving rise to satellite magnetic peaks in the reciprocal-space correlation function. It is clear that the basic $G$-type antiferromagnetic order is superimposed with the presence of an incommensurate structure with well-defined periodicity. The magnetic moments are found to rotate in the propagation plane, which is a defining character of a cycloid. A slight degree of anharmonicity of the cycloid in the small simulations explains the presence of weaker high-order harmonics in the correlation function [12]. The calculated magnetic hysteresis loop passes through the zero point and shows no remnant magnetization, giving a negligible weak-ferromagnetic behavior for the undoped case, although a metamagnetic transition is apparent at higher fields. This shows that the model Hamiltonian qualitatively reproduces
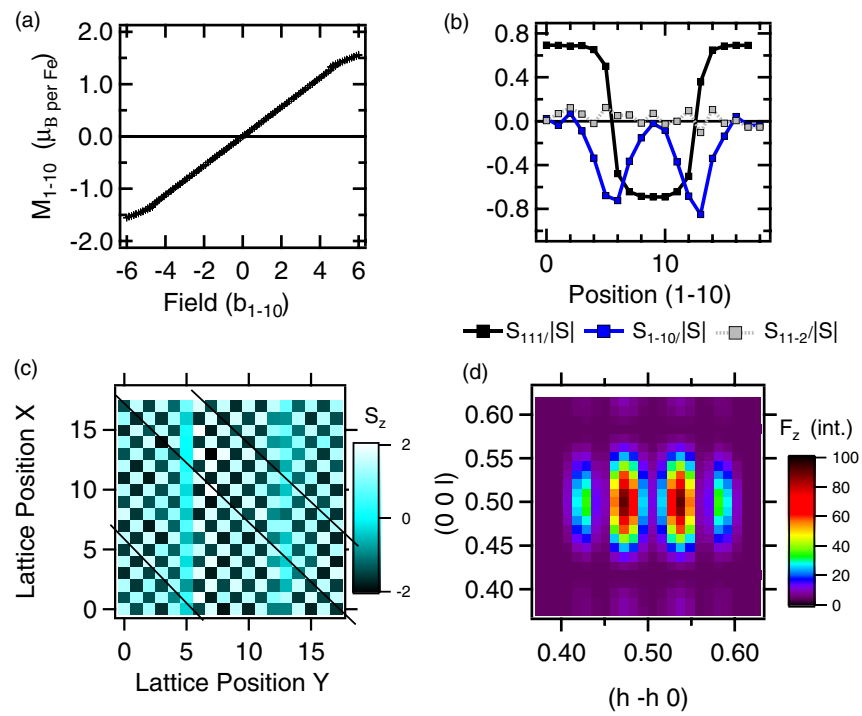

FIG. 6. (Color online) Results for the undoped case. (a) Hysteresis along the cycloid propagation (110). (b) Line profiles of spin orientation, revealing cycloidal propagation in the (111) direction. (c) Simulated lattice grid showing a cycloidal structure on top of antiferromagnetic order. (d) Simulated reciprocal-space map of the $\operatorname{AFM}\left(\frac{1}{2} \frac{1}{2} \frac{1}{2}\right)$ reflection

the key experimental magnetic properties known for undoped $\mathrm{BiFeO}_{3}$.

\section{Mn-doping behavior}

To model the effect of doping, we began by assuming that a new material with spin $(S=2.0)$ was substituted into the Fe-Fe $(S=2.5)$ lattice but the exchange constants were kept otherwise the same as those described in the previous section. Modifying the spin moment alone only results in a reduction of the average molecular field strengths by $\sim 20 \%$, which effectively scale as $\left\langle S^{2}\right\rangle$. In this case, a very similar correlation function and magnetic hysteresis to that shown for the undoped case in Fig. 6 was found. This indicates that simply reducing the average values of $\langle S\rangle$ to 2.25 through the local doping of $S=2$ spins at $50 \%$ concentration does not sufficiently perturb the model Hamiltonian to explain the removal of the cycloid as per the experiment. The result may be somewhat obvious because a spin-only modification preserves all ratios between the most important terms in the Hamiltonian which scale uniformly with $\left\langle|\vec{S}|^{2}\right\rangle$ such as $\left\langle|\vec{S}|^{2}\right\rangle \kappa,\left\langle|\vec{S}|^{2}\right\rangle D$, and $\left\langle|\vec{S}|^{2}\right\rangle J^{\prime}$ and $\left\langle|\vec{S}|^{2}\right\rangle J$, and it is the ratio of these terms which determine the turning angles and stability of the cycloid. Therefore, in order to drive the loss of the cycloid and weak-ferromagnetic behavior in the model system with doping, it is necessary to allow for modulation of the local molecular field ratios near a defect beyond a moderate universal scaling. In such a model, the presence of the $\mathrm{Mn}$ defect is assumed to alter the local environment in such a way that the relative ratios of $\kappa / J, D / J$, and $J^{\prime} / D$ are strongly affected in notably different ways. In this model, this was accounted for via the introduction of a new set of coupling constants: $J_{\mathrm{Fe}-\mathrm{Mn}}, J_{\mathrm{Mn}-\mathrm{Mn}}, J_{\mathrm{Mn}-\mathrm{Mn}}^{\prime}, J_{\mathrm{Fe}-\mathrm{Mn}}^{\prime}$, $D_{\mathrm{Fe}-\mathrm{Mn}}, D_{\mathrm{Fe}-\mathrm{Mn}}$, and $\kappa_{\mathrm{Mn}}$, which only apply for the bonds and sites occupied by a Mn defect. While past work examined a 
range of global magnetic parameters on the phase diagram of pure $\mathrm{BiFeO}_{3}[12,41]$, here we restrict the discussion to model only two types of local effects expected in the vicinity of a defect/dopant site in $\mathrm{Bi}_{0.8} \mathrm{La}_{0.2} \mathrm{Fe}_{0.5} \mathrm{Mn}_{0.5} \mathrm{O}_{3}$ :

(1) Simultaneous modification of the local superexchange constants $\left(J, J^{\prime}\right)$ together with the other local molecular field parameters (anisotropy and DM interaction) ( $\delta$-type perturbation).

(2) Modification of only the local anisotropy $\kappa$ while preserving the isotropic $J, J^{\prime}$ and anisotropic exchange $D$ constants ( $\epsilon$-type perturbation).

While the real system is undoubtedly more complicated than either of these two extremes, we will see that these modifications are useful test cases, and qualitatively reproduce the primary results of the experimental study. To this end, we note that the model presented in this study investigates the modified magnetic anisotropy of a magnetic defect site, and does not assess the potential electronic reconfiguration intrinsic to a $\mathrm{La} \mathrm{[11]} \mathrm{or} \mathrm{Mn}$ [23] dopant. The control over the molecular field parameters at a defect is implemented by writing the various parameters in terms of the original molecular field parameter appropriate for $\mathrm{BiFeO}_{3}$ scaled by perturbing variables $\delta$ and $\epsilon$ where the subscript 1 is applied to energies perturbing only $\mathrm{Mn}-\mathrm{Mn}$ pairs and factors affecting Fe-Mn pairs are notated with a 2:

$$
\begin{array}{ll}
J_{\mathrm{Mn}-\mathrm{Mn}}=\alpha_{1} \delta_{1} J_{\mathrm{Fe}-\mathrm{Fe}}, & J_{\mathrm{Mn}-\mathrm{Mn}}^{\prime}=\alpha_{1} \delta_{1} J_{\mathrm{Fe}-\mathrm{Fe}}^{\prime}, \quad \kappa_{\mathrm{Mn}}=\alpha_{1} \delta_{1} \epsilon \kappa_{\mathrm{Fe}}, \\
J_{\mathrm{Fe}-\mathrm{Mn}}=\alpha_{2} \delta_{2} J_{\mathrm{Fe}-\mathrm{Fe}}, \quad J_{\mathrm{Fe}-\mathrm{Mn}}^{\prime}=\alpha_{2} \delta_{2} J_{\mathrm{Fe}-\mathrm{Fe}}^{\prime} .
\end{array}
$$

In order to make the interpretation simpler, all of the energies involving $\mathrm{Mn}-\mathrm{Mn}$ pairs were rescaled by the spin ratios $\alpha_{1}=2.5^{2} / 2.0^{2}$ and the energies involving Fe-Mn pairs by $\alpha_{2}=2.5^{2} /(2.0 \times 2.5)$. In this case, the values of $\delta$ and $\epsilon$ just specify the net percentage of reduction or increase of the local molecular fields. Note that the values of $\mathrm{Fe}$ $\mathrm{Fe}$ parameters were fixed to those in the last column of Table II so that only regions in the matrix containing Mn had an altered environment. Although the orbital degeneracy of the $\mathrm{Mn}^{3+}$ ion in an octahedral symmetry configuration makes it Jahn-Teller active, it is known that even small doping concentrations $(5 \%-10 \%)$ of $\mathrm{Fe}$ on the $\mathrm{Mn}$ site in $\operatorname{LaMn}_{(1-x)} \mathrm{Fe}_{x} \mathrm{O}_{3}$ is enough to suppress Jahn-Teller distortions [45]. Considering the random placement of $\mathrm{Mn}$ ions in our system, we neglect these effects in the model to simplify calculations.

In this work, the simulation was set to scale the Fe-Mn and $\mathrm{Mn}-\mathrm{Mn}$ parameters together. This is notated without a subscript signifying that $\delta_{1}=\delta_{2}=\delta$. The results of Mn-doped behavior at $50 \%$ dilution for various strengths of the perturbing variables are shown in Fig. 7. For all $\delta \geqslant 0.5$ scalings, the lowtemperature cycloid ground state is preserved, whereas below this threshold long-range $G$-type antiferromagnetic order was stable down to $\delta=0.165$. Figure 8 (right) shows the effects of varying the local anisotropy for the Mn dopants on the spincorrelation functions. Unlike the case for a $\delta$-type perturbation, where a lower limit for cycloid formation is seen, for a local anisotropy $\epsilon$-type perturbation the cycloid only forms in an intermediary region corresponding to where $\epsilon \approx 1$ (i.e., an anisotropy similar to the unperturbed case). This result shows that an effective route to replace the cycloid with a $G$-type
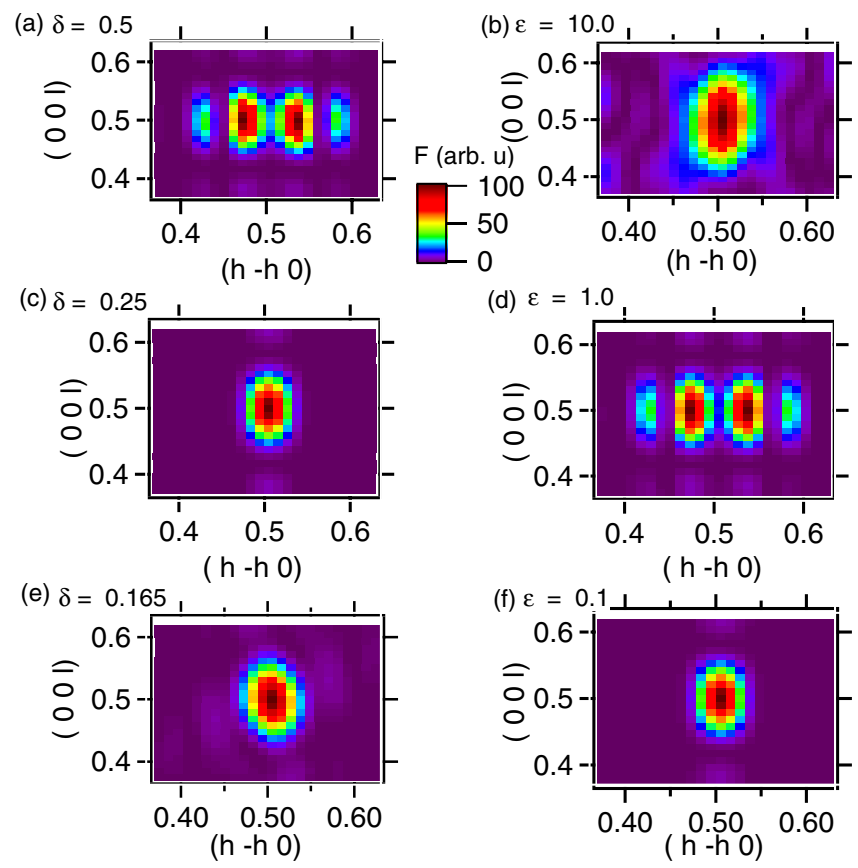

FIG. 7. (Color online) Reciprocal-space maps of the antiferromagnetic $\left(\frac{1}{2} \frac{1}{2} \frac{1}{2}\right)$ reflection, illustrating the effect of varying [(a), (c), (e)] $\delta$-type and [(b), (d), (f)] $\epsilon$-type perturbations upon the spin structure. Whereas the $\delta$ type features a cycloid threshold minimum of 0.5 , the $\epsilon$ type features an intermediary region around 1.0.

AFM ground state appears to be the decrease or increase of the ratio of local anisotropy to the superexchange constants. Past work discussed the collapse of the cycloid under high anisotropy [12], but here we show that there is also a lower threshold below which the cycloid appears to be unstable in the vicinity of a magnetic defect.

Figure 8 shows the magnetic hysteresis for various increases and decreases to the local anisotropy. For increased local anisotropy near a defect with $\epsilon=10$, a clear weakferromagnetic behavior is evident in the calculated hysteresis loops. Together with the correlation functions, this result shows that even when the modification of the local anisotropy near a defect is sufficient to cause the collapse of the cycloid, it does

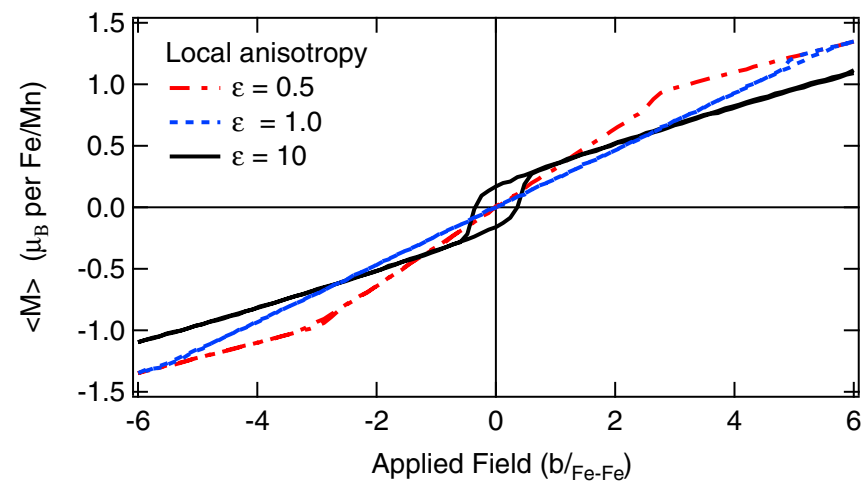

FIG. 8. (Color online) Effect of increasing the anisotropy pertubation upon the simulated magnetic hysteresis at $50 \% \mathrm{Mn}$ substitution. 

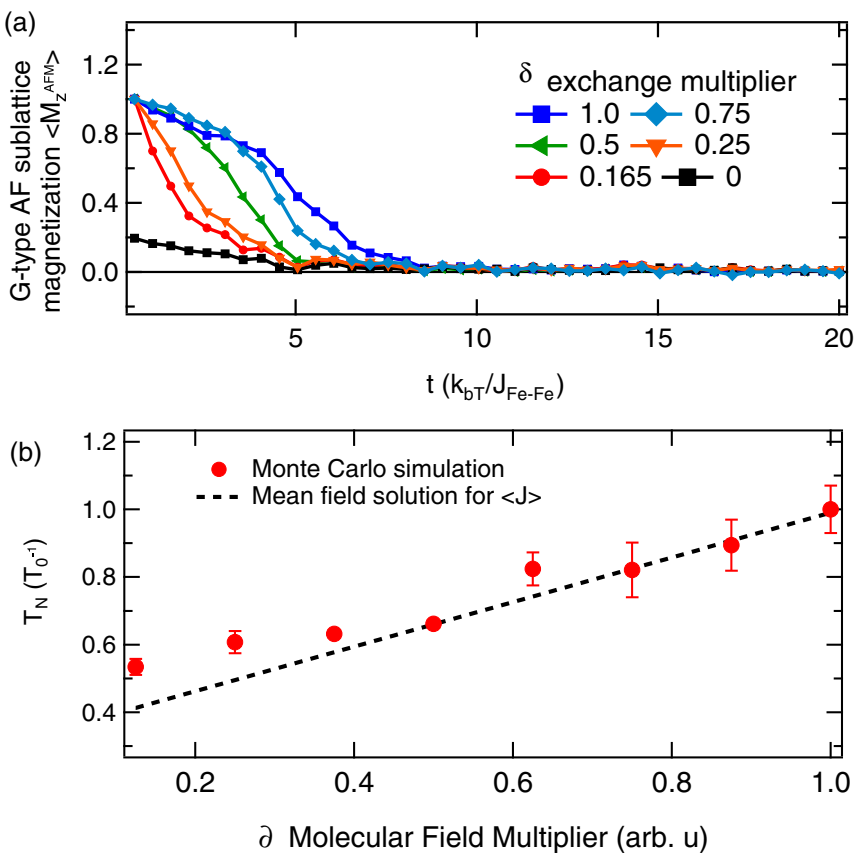

FIG. 9. (Color online) (a) Temperature dependency of AFM Gtype staggered magnetization with increasing $\delta$-type perturbation strength. (b) A mean-field treatment matches well with our results for low to moderate perturbations, but for stronger perturbations it fails to provide realistic results.

not always lead to weak-ferromagnetic hysteresis. In Fig. 9(a), the effect of reducing $\delta$ on the transition temperature in the system is shown. To check the robustness of this result near the percolation threshold, the number of MCs in the simulation per temperature interval was varied between 100 and 10000 , however, similar results were found. It is clear that a local reduction of the entire set of molecular field parameters in the region of a $\mathrm{Mn}$ atom reduces the critical temperature, even when the remaining $\mathrm{Fe}-\mathrm{Fe}$ interactions are unaltered and, while magnetic preorder may exist in the model, there is predominantly a single transition. The inclusion of dopants that reduce the antiferromagnetic interactions will obviously reduce the average Néel temperature. For the $x=0.5$ concentration, equal numbers of $\mathrm{Fe}-\mathrm{Fe}, \mathrm{Mn}-\mathrm{Mn}$, and $\mathrm{Mn}-\mathrm{Fe}$ pairs are formed in the system. This suggests that the mean primary exchange in the system is

$$
\langle J\rangle=\frac{J_{\mathrm{Fe}-\mathrm{Fe}}+J_{\mathrm{Mn}-\mathrm{Fe}}+J_{\mathrm{Mn}-\mathrm{Mn}}}{3} .
$$

Using this mean-field approach, the Néel temperature is proportional to $\langle J\rangle$ such that if one defines a parameter such as $\delta$ that modifies both $J_{\mathrm{Mn}-\mathrm{Mn}}$ and $J_{\mathrm{Mn}-\mathrm{Fe}}$, then

$$
T_{N}(\delta) / T_{0}=\frac{2}{3} \delta+\frac{1}{3} .
$$

Thus, the simple mean-field treatment suggests that a decrease in $\delta$ will lower the Néel temperature linearly for $50 \%$ concentration. Figure 9 (b) compares the result of decreasing $\delta$ with the mean-field solution. The mean-field result is approximately obeyed for low to moderate perturbations. However, for stronger perturbations, corresponding to a lower $\delta$, the relation does not hold well. Unlike the
TABLE III. Energy ratios used in the simulation of disordered $\mathrm{BiMn}_{0.5} \mathrm{Fe}_{0.5} \mathrm{O}_{3}$ in terms of $J_{\mathrm{FM}-\mathrm{FM}}$. In simulation (1), relative ratios of $J^{\prime}, \kappa$, and $D$ to the primary constant $J_{\mathrm{Fe}-\mathrm{Fe}}$ were implemented to enable potential reproduction of the cycloid within the size limits of the simulation. In simulation (2), the absolute literature values were used.

\begin{tabular}{lcc}
\hline \hline Parameter & Simulation (1) & Simulation (2) \\
\hline$J_{\mathrm{Fe}-\mathrm{Fe}}$ & -1.0 & -1.0 \\
$J_{\mathrm{Fe}-\mathrm{Fe}}^{\prime}$ & $-0.18 / J_{\mathrm{Fe}-\mathrm{Fe}}$ & $-0.034 / J_{\mathrm{Fe}-\mathrm{Fe}}$ \\
$\kappa_{\mathrm{Fe}}$ & $0.08 / J_{\mathrm{Fe}-\mathrm{Fe}}$ & $0.001 / J_{\mathrm{Fe}-\mathrm{Fe}}$ \\
$D_{\mathrm{Fe}}$ & $0.144 / J_{\mathrm{Fe}-\mathrm{Fe}}$ & $0.024 / J_{\mathrm{Fe}-\mathrm{Fe}}$ \\
$J_{\mathrm{Mn}-\mathrm{Fe}}=J_{\mathrm{Mn}-\mathrm{Mn}}$ & $-0.174 / J_{\mathrm{Fe}-\mathrm{Fe}}$ & $-0.174 / J_{\mathrm{Fe}-\mathrm{Fe}}$ \\
$J_{\mathrm{Mn}-\mathrm{Fe}}^{\prime}=J_{\mathrm{Mn}-\mathrm{Mn}}^{\prime}$ & $-0.18 / J_{\mathrm{Fe}-\mathrm{Fe}}$ & $-0.23 / J_{\mathrm{Fe}-\mathrm{Fe}}$ \\
$\kappa_{\mathrm{Mn}}$ & $0.08 / J_{\mathrm{Fe}-\mathrm{Fe}}$ & $0.01 / J_{\mathrm{Fe}-\mathrm{Fe}}$ \\
$D_{\mathrm{Mn}-\mathrm{Fe}}=D_{\mathrm{Mn}-\mathrm{Mn}}$ & $0.2 / J_{\mathrm{Fe}-\mathrm{Fe}}$ & $0.068 / J_{\mathrm{Fe}-\mathrm{Fe}}$ \\
\hline \hline
\end{tabular}

numerical simulation, the mean-field model suggests that even in the situation $J_{\mathrm{Mn}-\mathrm{Mn}}=J_{\mathrm{Mn}-\mathrm{Fe}}=0$, antiferromagnetic order will be mediated by $\mathrm{Fe}-\mathrm{Fe}$ pairs, with a $T_{\mathrm{N}} \approx$ $\frac{1}{3} T_{0}$. This is unphysical near the percolation threshold as short-range order is formed in a increasingly paramagnetic matrix.

\section{Comparison with experiment for $\mathrm{Bi}_{0.8} \mathrm{La}_{0.2} \mathrm{Fe}_{0.5} \mathrm{Mn}_{0.5} \mathrm{O}_{3}$}

The results of the previous section indicate that numerous types of perturbations can trigger the collapse of the cycloid in $\mathrm{Bi}_{0.8} \mathrm{La}_{0.2} \mathrm{Fe}_{0.5} \mathrm{Mn}_{0.5} \mathrm{O}_{3}$ presented in this study. While no detailed DFT calculations have been performed for disordered $\mathrm{Bi}_{0.8} \mathrm{La}_{0.2} \mathrm{Fe}_{0.5} \mathrm{Mn}_{0.5} \mathrm{O}_{3}$, using appropriate terms based on similar studies of related materials we can estimate molecular field parameters to derive a strong model. We have adopted approximate values for the $J_{\mathrm{Mn}-\mathrm{Mn}}, J_{\mathrm{Mn}-\mathrm{Mn}}^{\prime}, \kappa_{\mathrm{Mn}}$, and $D_{\mathrm{Mn}-\mathrm{Mn}}$ terms based upon calculations for $R E \mathrm{MnO}_{3}$ [23], and note that the superexchange in that case is also compatible with the transition temperature also reported experimentally for $\mathrm{BiMnO}_{3}$ in the high-pressure AFM state [46]. For our simulations, we assume that reasonably similar values apply for Fe-Mn interactions, which is supported by the almost linear dependence of critical temperature on the substitution level in $\mathrm{BiMn}_{x} \mathrm{Fe}_{1-x} \mathrm{O}_{3}$ compounds [5], and the relative insensitivity of the transition temperature to compositional differences of $\mathrm{Mn}$ and $\mathrm{Fe}$ near 50\% concentration in related materials [47]. Simulations were performed with two energy regimes as listed in Table III. For simulation 1, relative ratios of $D / J^{\prime}, \kappa / J^{\prime}$, and $J / J^{\prime}$ are preserved to the approximate values for $\mathrm{BiFeO}_{3}$ and $R E \mathrm{MnO}_{3}$, but $J^{\prime}$ is increased to values appropriate for the potential formation of a cycloid on a $18 \times 18$ lattice used for this calculation to ensure that any collapse of the cycloid is not a finite-size effect of having a large cycloid in a small simulation volume. Simulation 2 applies absolute versions of the energies based on the known values for $\mathrm{BiFeO}_{3}$ and $R E \mathrm{MnO}_{3}$. Figure 10 presents the results of both simulations. In both cases, the simulation mirrors experimental results for the following key findings: (1) the suppression of the cycloid, (2) a marked reduction in the Néel temperature, and (3) the enhancement of a weak-ferromagnetic moment, associated with coherent canting. The shape of 

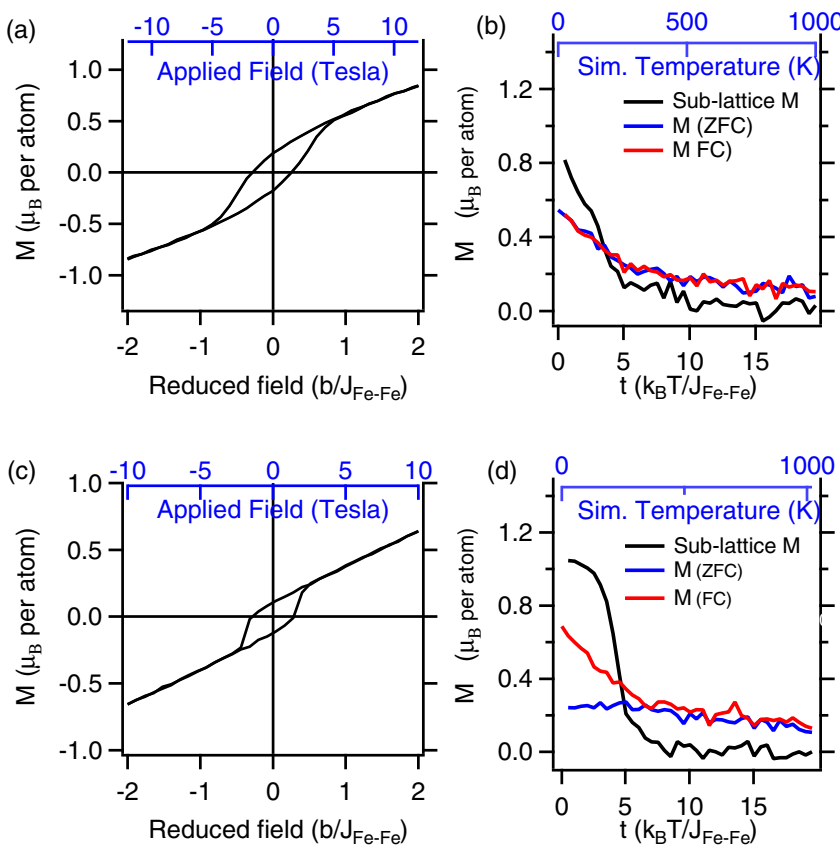

FIG. 10. (Color online) (a) Simulated hysteresis and (b) magnetic transition for relative experimental energies for simulation 2 which, in absence of Mn doping, give a cycloid length of 18 unit cells. (c) Simulation 1 hysteresis and (d) magnetic transition for absolute energy ratios. The simulation units have been rescaled assuming $\left|\overrightarrow{S_{\mathrm{Fe}}}\right|=2.5$ and $\left|J_{\mathrm{Fe}-\mathrm{Fe}}\right|=4.38 \mathrm{meV}=50 \mathrm{~K}=76 \mathrm{~T} / \mathrm{gS}$.

the hysteresis is close to the experimental case presented in Fig. 2 and the saturation magnetic moment from the weak-ferromagnetic contribution is $\sim 0.5 \mu_{B}$, in agreement with our experimental observations. With the absolute energy values of simulation 2 , the magnetic transition temperature was determined to be $T_{N} \approx 270 \mathrm{~K}$, which is comparable to our experimentally determined value of $240 \mathrm{~K}$. Moreover, in Fig. 10(b) bifurcation is apparent in the field-cooled and zero-field-cooled $M$ versus $T$ trends, directly comparable to our experimental results in Fig. 2. In our model, we note that bifurcation is only present when weak-ferromagnetic order leads to a modification of the antiferromagnetic Curie-Weiss law. The bifurcation point in the model coincides with $T_{N}$, whereas in our experimental results it lies slightly below the transition. This could indicate a magnetic-field dependence in the experiment or an additional spin reorientation that is not captured in the model. It should be pointed out that while both simulations give similar results, they employ quite different absolute values of the Mn anisotropy and the DM interaction. As such, it is clear that a common factor driving the magnetic modifications is the reduced superexchange constants at the defect site $\left(J_{\mathrm{Mn}-\mathrm{Mn}}, J_{\mathrm{Fe}-\mathrm{Mn}}\right)$, and it is not the absolute values of the other parameters that is crucial, but their ratio with respect to the primary local exchange. Based on the related compounds, it seems that the well-established weakness of $\mathrm{Mn}-\mathrm{O}-\mathrm{Mn}$ superexchange $J_{\mathrm{Mn}-\mathrm{Mn}}$ compared to the $\mathrm{Fe}-\mathrm{O}-\mathrm{Fe}$ bond in $\mathrm{BiFeO}_{3}\left(J_{\mathrm{Fe}-\mathrm{Fe}}\right)$ is likely responsible for both the reduced transition temperature in $\mathrm{Bi}_{0.8} \mathrm{La}_{0.2} \mathrm{Fe}_{0.5} \mathrm{Mn}_{0.5} \mathrm{O}_{3}$ and for modifying other relevant ratios to a point where the cycloid is not stable. While it is likely that modification of the DM interaction and the anisotropy also occur near a defect, our result implies that this is hardly necessary to aptly describe the cycloid collapse and emergent wFM. Even assuming electronic similarity between $\mathrm{Fe}$ and $\mathrm{Mn}$ in other respects, the superexchange modification due to the introduction of a magnetic defect is likely the primary driver in a homogeneous compound.

\section{CONCLUSIONS}

Neutron diffraction and magnetometry studies of polycrystalline $\mathrm{Bi}_{(1-x)} \mathrm{La}_{(x)} \mathrm{Fe}_{(1-y)} \mathrm{Mn}_{(y)} \mathrm{O}_{3}$ with $x=0.2$ and $y=0.5$ indicated the system stabilizes in the Imma orthorhombic perovskite crystallographic symmetry down to lowest temperatures, with a transition to AFM order at $T_{N} \approx 240 \mathrm{~K}$ and a suppression of the spin-cycloid structure. In comparison to the calculated properties of undoped $\mathrm{BiFeO}_{3}$, the $\mathrm{Bi}_{0.8} \mathrm{La}_{0.2} \mathrm{Fe}_{0.5} \mathrm{Mn}_{0.5} \mathrm{O}_{3}$ system possesses an enhanced magnetic susceptibility and WFM component, despite relatively similar crystallographic structure with the oxygen-tilting angle determined to be almost identical. As such, the improved magnetic performance likely arises from fundamental perturbations of electron exchange interactions due to the inclusion of $\mathrm{Mn}$ ions on the Fe-ion B sites, which we studied in detail with a classical microstructural spin Hamiltonian model implemented via a Monte Carlo computer simulation. Despite its relative simplicity, the classical vector-spin model approach enables a larger scale investigation and, importantly, qualitatively reproduces the main physical observables found for the case of bulk $\mathrm{BiFeO}_{3}, \mathrm{Bi}_{0.8} \mathrm{La}_{0.2} \mathrm{Fe}_{0.5} \mathrm{Mn}_{0.5} \mathrm{O}_{3}$, and thinfilm-form $\mathrm{BiMn}_{0.5} \mathrm{Fe}_{0.5} \mathrm{O}_{3}$ [27]. The reduced Néel temperature in $\mathrm{BiMn}_{0.5} \mathrm{Fe}_{0.5} \mathrm{O}_{3}$ is primarily attributed to the reduction of average primary nearest-neighbor exchange forces due to the bonds formed in $\mathrm{Mn}-\mathrm{Fe}$ and $\mathrm{Mn}-\mathrm{Mn}$ regions. The cycloidal ground state and weak-ferromagnetic behavior are intricately connected. The eradication of the cycloid is a necessary condition for the appearance of a notable ferromagnetic component, but does not guarantee its occurrence. In particular, the local modification of the ratios of the anisotropy $\kappa / J$ is an efficient route to driving the system to a weak-ferromagnetic state. If spin-orbit anisotropy and superexchange for the $\mathrm{Mn}$ sites in the $\mathrm{Bi}_{0.8} \mathrm{La}_{0.2} \mathrm{Fe}_{0.5} \mathrm{Mn}_{0.5} \mathrm{O}_{3}$ is of a similar magnitude to related Mn compounds, then this provides the explanation for the rise of weak ferromagnetism. Nevertheless, first-principles determinations of the Fe-impurity exchange parameters in doped $\mathrm{BiFeO}_{3}$ systems are urgently required for understanding of the optimum dopant and concentration via a multiscale modeling approach.

\section{ACKNOWLEDGMENTS}

D.L.C. and J.B. acknowledge the support of the Australian Institute of Nuclear Science and Engineering Inc. (AINSE). X.L.W., F.K., J.B., and C.U. acknowledge the support of the Australian Research Council (ARC) through the Discovery Projects funding scheme (Projects No. DP0987190 and No. DP110105346). Z. X. Cheng thanks the ARC for support through a Future Fellowship. 
[1] M. Bibes and A. Barthélémy, Nat. Mater. 7, 425 (2008).

[2] H. Béa, M. Gajek, M. Bibes, and A. Barthélémy, J. Phys.: Condens. Matter 20, 434221 (2008).

[3] T. Choi, S. Lee, Y. J. Choi, V. Kiryukhin, and S.-W. Cheong, Science 324, 63 (2009).

[4] G. Catalan and J. F. Scott, Adv. Mater. 21, 2463 (2009).

[5] I. Sosnowska, W. Schäfer, W. Kockelmann, K. Andersen, and I. Troyanchuk, Appl. Phys. A: Mater. Sci. Process. 74, S1040 (2002).

[6] J.-H. Lee, H. J. Choi, D. Lee, M. G. Kim, C. W. Bark, S. Ryu, M.-A. Oak, and H. M. Jang, Phys. Rev. B 82, 045113 (2010).

[7] Z. X. Cheng, A. H. Li, X. L. Wang, S. X. Dou, K. Ozawa, H. Kimura, S. J. Zhang, and T. R. Shrout, J. Appl. Phys. 103, 07E507 (2008).

[8] S. R. Das, R. N. P. Choudhary, P. Bhattacharya, R. S. Katiyar, P. Dutta, A. Manivannan, and M. S. Seehra, J. Appl. Phys. 101, 034104 (2007).

[9] R. Sæterli, S. M. Selbach, P. Ravindran, T. Grande, and R. Holmestad, Phys. Rev. B 82, 064102 (2010).

[10] M. Azuma, H. Kanda, A. A. Belik, Y. Shimakawa, and M. Takano, J. Magn. Magn. Mater. 310, 1177 (2007).

[11] C. Weingart, N. A. Spaldin, and E. Bousquet, Phys. Rev. B 86, 094413 (2012).

[12] D. Rahmedov, D. Wang, J. Íniguez, and L. Bellaiche, Phys. Rev. Lett. 109, 037207 (2012).

[13] J. Jeong, E. A. Goremychkin, T. Guidi, K. Nakajima, G. S. Jeon, S.-A. Kim, S. Furukawa, Y. B. Kim, S. Lee, V. Kiryukhin, S.-W. Cheong, and J.-G. Park, Phys. Rev. Lett. 108, 077202 (2012).

[14] M. Matsuda, R. S. Fishman, T. Hong, C. H. Lee, T. Ushiyama, Y. Yanagisawa, Y. Tomioka, and T. Ito, Phys. Rev. Lett. 109, 067205 (2012).

[15] I. Dzyaloshinksy, J. Phys. Chem. Solids 4, 241 (1958).

[16] T. Moriya, Phys. Rev. 120, 91 (1960).

[17] C. Ederer and N. A. Spaldin, Phys. Rev. B 71, 060401 (2005).

[18] I. Sosnowska, T. P. Neumaier, and E. Steichele, J. Phys. C: Solid State Phys. 15, 4835 (1982).

[19] D. Lebeugle, D. Colson, A. Forget, M. Viret, A. M. Bataille, and A. Goukasov, Phys. Rev. Lett. 100, 227602 (2008).

[20] S. Lee, W. Ratcliff, S.-W. Cheong, and V. Kiryukhin, Appl. Phys. Lett. 92, 192906 (2008).

[21] M. Ramazanoglu, M. Laver, W. Ratcliff, S. M. Watson, W. C. Chen, A. Jackson, K. Kothapalli, S. Lee, S.-W. Cheong, and V. Kiryukhin, Phys. Rev. Lett. 107, 207206 (2011).

[22] J. T. Zhang, X. M. Lu, J. Zhou, H. Sun, J. Su, C. C. Ju, F. Z. Huang, and J. S. Zhu, Appl. Phys. Lett. 100, 242413 (2012).

[23] M. Mochizuki and N. Furukawa, Phys. Rev. B 80, 134416 (2009).

[24] R. Haumont, P. Bouvier, A. Pashkin, K. Rabia, S. Frank, B. Dkhil, W. A. Crichton, C. A. Kuntscher, and J. Kreisel, Phys. Rev. B 79, 184110 (2009).
[25] F. Bai, J. Wang, M. Wuttig, J. Li, N. Wang, A. P. Pyatakov, A. K. Zvezdin, L. E. Cross, and D. Viehland, Appl. Phys. Lett. 86, 032511 (2005).

[26] H. Zhao, H. Kimura, Q. Yao, Y. Du, Z. Cheng, and X. Wang, in Ferroelectrics-Material Aspects, edited by M. Lallart (InTech, Janeza Trdine, 2011), pp. 237-258.

[27] D. L. Cortie, A. P. J. Stampfl, F. Klose, Y. Du, X. L. Wang, H. Y. Zhao, H. Kimura, and Z. X. Cheng, Appl. Phys. Lett. 101, 172404 (2012).

[28] J. Rodrguez-Carvajal, Phys. B (Amsterdam) 192, 55 (1993).

[29] D. C. Arnold, K. S. Knight, F. D. Morrison, and P. Lightfoot, Phys. Rev. Lett. 102, 027602 (2009).

[30] A. M. Glazer, Acta Crystallogr., Sect. B: Struct. Crystallogr. Cryst. Chem. 28, 3384 (1972).

[31] B. J. Kennedy, C. J. Howard, and B. C. Chakoumakos, J. Phys.: Condens. Matter 11, 1479 (1999).

[32] H. D. Megaw and C. N. W. Darlington, Acta Crystallogr., Sect. A: Cryst. Phys., Diffr., Theor. Gen. Crystallogr. 31, 161 (1975).

[33] D. Y. Wang, N. Y. Chan, R. K. Zheng, C. Kong, D. M. Lin, J. Y. Dai, H. L. W. Chan, and S. Li, J. Appl. Phys. 109, 114105 (2011).

[34] D. E. C. P. Thompson and J. B. Hastings, J. Phys.: Condens. Matter 20, 79 (1987).

[35] I. Sosnowska, M. Loewenhaupt, W. David, and R. M. Ibberson, Mater. Sci. Forums 133-136, 683 (1993).

[36] C. A. Bridges, A. S. Sefat, E. A. Payzant, L. Cranswick, and M. P. Paranthaman, J. Solid State Chem. 184, 830 (2011).

[37] A. Wills, Phys. B (Amsterdam) 276, 680 (2000).

[38] J. R. Sahu and C. Rao, Solid State Sci. 9, 950 (2007).

[39] J. A. M. Cagigas, D. S. Candela, and E. Baggio-Saitovitch, J. Phys.: Conf. Ser. 200, 012134 (2010).

[40] D. A. Rusakov, A. M. Abakumov, K. Yamaura, A. A. Belik, G. Van Tendeloo, and E. Takayama-Muromachi, Chem. Mater. 23, 285 (2011).

[41] D. L. Cortie, Ph.D. thesis, University of Wollongong, Australia, 2013.

[42] U. Nowak, in Handbook of Magnetism and Advanced Magnetic Materials, Vol. 1, edited by H. Kronmüller and S. Parkin (Wiley, New York, 2007).

[43] U. Nowak, K. D. Usadel, J. Keller, P. Miltenyi, B. Beschoten, and G. Güntherodt, Phys. Rev. B 66, 014430 (2002).

[44] L. Bi, A. R. Taussig, H.-S. Kim, L. Wang, G. F. Dionne, D. Bono, K. Persson, G. Ceder, and C. A. Ross, Phys. Rev. B 78, 104106 (2008).

[45] D. V. Karpinsky, I. O. Troyanchuk, and V. V. Sikolenko, J. Phys.: Condens. Matter 19, 036220 (2007).

[46] D. P. Kozlenko, A. A. Belik, S. E. Kichanov, I. Mirebeau, D. V. Sheptyakov, T. Strässle, O. L. Makarova, A. V. Belushkin, B. N. Savenko, and E. Takayama-Muromachi, Phys. Rev. B 82, 014401 (2010).

[47] O. F. de Lima, J. A. H. Coaquira, R. L. de Almeida, L. B. de Carvalho,, and S. K. Malik, J. Appl. Phys. 105, 013907 (2009). 